\title{
Resveratrol attenuates neuronal autophagy and inflammatory injury by inhibiting the TLR4/NF-кB signaling pathway in experimental traumatic brain injury
}

\author{
YAN FENG ${ }^{1}$, YING CUI ${ }^{2}$, JUN-LING GAO ${ }^{3,4}$, MING-HANG LI ${ }^{3}$, RAN LI $^{3,4}$, XIAO-HUA JIANG ${ }^{3,4}$, \\ YAN-XIA TIAN $^{3,4}$, KAI-JIE WANG ${ }^{2}$, CHANG-MENG CUI $^{1}$ and JIAN-ZHONG CUI ${ }^{1,2}$ \\ ${ }^{1}$ Department of Surgery, Hebei Medical University, Shijiazhuang, Hebei 050017; \\ ${ }^{2}$ Department of Neurosurgery,Tangshan Workers' Hospital; ${ }^{3}$ School of Basic Medical Science, \\ Hebei United University; ${ }^{4}$ Hebei Key Laboratory for Chronic Diseases, Tangshan Key Laboratory \\ for Preclinical and Basic Research on Chronic Diseases, Tangshan, Hebei 063000, P.R. China
}

Received June 3, 2015; Accepted February 8, 2016

DOI: $10.3892 / \mathrm{ijmm} .2016 .2495$

\begin{abstract}
Previous research has demonstrated that traumatic braininjury (TBI) activates autophagy and a neuroinflammatory cascade that contributes to substantial neuronal damage and behavioral impairment, and Toll-like receptor 4 (TLR4) is an important mediator of this cascade. In the present study, we investigated the hypothesis that resveratrol $(\mathrm{RV})$, a natural polyphenolic compound with potent multifaceted properties, alleviates brain damage mediated by TLR4 following TBI. Adult male Sprague Dawley rats, subjected to controlled cortical impact (CCI) injury, were intraperitoneally injected with RV (100 mg/kg, daily for 3 days) after the onset of TBI. The results demonstrated that RV significantly reduced brain edema, motor deficit, neuronal loss and improved spatial cognitive function. Double immunolabeling demonstrated that RV decreased microtubule-associated protein 1 light chain 3 (LC3), TLR4-positive cells co-labeled with the hippocampal neurons, and RV also significantly reduced the number of TLR4-positive neuron-specific nuclear protein (NeuN) cells following TBI. Western blot analysis revealed that RV significantly reduced the protein expression of the autophagy marker proteins, LC3II and Beclin1, in the hippocampus compared with that in the TBI group. Furthermore, the levels of TLR4 and its known downstream signaling molecules, nuclear factor- $\kappa \mathrm{B}(\mathrm{NF}-\kappa \mathrm{B})$,
\end{abstract}

Correspondence to: Dr Jian-Zhong Cui, Department of Surgery, Hebei Medical University, 361 East Zhongshan Road, Shijiazhuang, Hebei 050017, P.R. China

E-mail: jzhcuidr2001@163.com

Abbreviations: RV, resveratrol; TLR4, Toll-like receptor 4; TBI, traumatic brain injury; LC3, microtubule-associated protein 1 light chain 3; NeuN, neuron-specific nuclear protein; DAPI, 4',6-diamidino-2-phenylindole; NSS, neurologic severity score

Key words: resveratrol, autophagy, traumatic brain injury, Toll-like receptor 4 , inflammation and the inflammatory cytokines, interleukin (IL)-1 $\beta$ and tumor necrosis factor (TNF)- $\alpha$ were also decreased after $\mathrm{RV}$ treatment. Our results suggest that RV reduces neuronal autophagy and inflammatory reactions in a rat model of TBI. Thus, we suggest that the neuroprotective effect of RV is

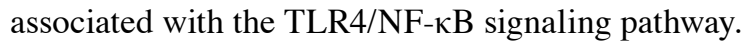

\section{Introduction}

Traumatic brain injury (TBI) is a highly complex type of neurological trauma that is caused by both primary and secondary brain injury mechanisms, and the latter plays a crucial role in the clinical outcome of patients with TBI (1). Owing to their potential to be effectively treated using post-injury therapeutic intervention, understanding the mechanisms underlying secondary brain injury is essential. In particular, a complex series of sterile inflammatory responses play an important role in the development of secondary brain injury following TBI $(2,3)$, and the mechanisms regulating this process remain poorly understood. Thus, the inhibition of proinflammatory mediators offers a potentially effective therapeutic approach for TBI.

Toll-like receptors (TLRs) are transmembrane proteins that play a major role in the recognition of pathogen-associated molecular patterns present on viral and bacterial products (4). Among these TLRs, Toll-like receptor 4 (TLR4) has been shown to play an important role in initiating the inflammatory response and ultimately leading to inflammatory injury and neurological deficits following stroke or head trauma (5-7). Furthermore, the recruitment of myeloid differentiation factor 88 (MyD88), a critical adapter protein for TLR4, leads to the activation of downstream nuclear factor $-\kappa \mathrm{B}(\mathrm{NF}-\kappa \mathrm{B})$ and the subsequent production of proinflammatory cytokines implicated in neurotoxicity $(8,9)$.

Autophagy is an evolutionarily conserved pathway that leads to the degradation of proteins and entire organelles in cells undergoing stress (10). Although autophagy involves a stress adaptation pathway that promotes cell survival under most circumstances, previous research has demonstrated that this pathway can trigger cell injury and death under certain 
pathological circumstances (11). Previous research, and our previous study, has demonstrated that the autophagy pathway is involved in the pathophysiological responses following TBI, and inhibition of this pathway may attenuate traumatic damage and functional outcome deficits (12-14). Autophagy has been shown to be an important component of the innate immune response, and TLR4 serves as an environmental sensor for autophagy (15-17). However, in TBI-induced inflammatory injury, the role of TLR4 in neuronal autophagy and activation remains unclear. Therefore, in the present study, we investigated the role of TLR4 in neuronal autophagy and activation in TBI-induced inflammatory injury.

Resveratrol (3,5,4'-trihydroxystilbene, RV) occurs naturally in grapes and a variety of medicinal plants, and abundant evidence has demonstrated that RV may be a candidate for TBI therapy. The neuroprotective effects of RV in central nervous system injuries are associated with its antioxidant (18), anti-inflammatory (19), anti-apoptotic properties (20), as well as reduced autophagy (21). A previous study has demonstrated that RV exerts a protective effect in cardiomyocytes by suppressing the TLR4/NF-KB signaling pathway (22). Another report has shown that $\mathrm{RV}$ exerts a protective effect against the interleukin (IL)-1 $\beta$-induced inflammatory responses on human osteoarthritic chondrocytes partly through the TLR4/MyD88/ NF- $\kappa B$ signaling pathway (23). However, the protective effect of RV and the mechanisms through which it exerts this effect following TBI require further examination. In addition, it is necessary to determine whether RV is capable of modulating TBI through the TLR4/NF- $\kappa B$ signaling pathway and downregulation of neuronal autophagy.

In the present study, we examined the effect of $\mathrm{RV}$ on post-TBI brain edema, spatial cognitive function and neurological impairment in a rat model of TBI. At $24 \mathrm{~h}$ post-injury, the activation of microtubule-associated protein 1 light chain 3 (LC3), TLR4 and neuron-specific nuclear protein (NeuN) was detected by immunofluorescence; the downstream signaling molecules of TLR4 and autophagy-related proteins were also assessed by western blot analysis. The results provide new evidence for RV-mediated neuroprotection in a rat model of TBI.

\section{Materials and methods}

Establishment of a rat model of TBI. A total of 170 adult male Sprague-Dawley rats (weighing 300-330 g) were obtained from the Hebei United University Experimental Animal Center (Tangshan, China). The animals were housed, and a 12-h light/dark cycle was applied, and water and food were provided ad libitum prior to and following surgery or the sham operation. All experiments were approved by the Ethics Committee of Hebei United University for the use of animals. A previously described controlled cortical impact (CCI) rat model of TBI was utilized for this study (24). Briefly, the rats were intraperitoneally anesthetized with $10 \%$ chloral hydrate $(3 \mathrm{ml} / \mathrm{kg})$ and placed in a stereotaxic frame. Utilizing aseptic techniques, a midline incision was made to expose the skull between the bregma and lambda suture lines. A 6-mm craniotomy was performed over the right parietal cortex, centered on the coronal suture and $3 \mathrm{~mm}$ lateral to the sagittal suture. The underlying dura mater was kept intact over the cortex. A cortical contusion was produced using a rounded metal tip (4-mm diameter) which was positioned at the center of the craniotomy and lowered over the craniotomy site until it touched the dura mater. A velocity of $5 \mathrm{~m} / \mathrm{sec}$ and a deformation depth of $2.5 \mathrm{~mm}$ below the dura were used. The bone flap was immediately replaced and sealed, and the scalp was sutured closed. The rats were housed in individual cages following surgery and placed on heat pads $\left(37^{\circ} \mathrm{C}\right)$ for $24 \mathrm{~h}$ to maintain normal body temperature during the recovery period. The sham-operated animals were anesthetized and underwent a craniotomy as described above, without undergoing CCI.

Groups and drug administration. A total of 170 rats were used in this study. The rats were randomly divided into three groups $(n=5$ at each time point): sham-operated group $(n=50)$; TBI group $(n=60)$; and TBI in combination with RV group $(n=60)$. Of the total number of rats that underwent TBI and TBI in combination with RV, 16 rats died of trauma, and were eliminated from subsequent experiments. RV (Sigma-Aldrich, Yorba Linda, CA, USA) was freshly prepared by dissolving it in 50\% ethanol and diluting it in $0.9 \%$ saline at a concentration of $100 \mathrm{mg} / \mathrm{kg}$, and was administered bydaily intraperitoneal injection to the rats in the RV groups for 3 days, beginning immediately after TBI, as previously described (14). Both the sham-operated and TBI groups received equal volumes of ethanol (2\%) by intraperitoneal injection at the same time daily. All investigations were blind and the animal codes were revealed only at the end of the behavioral and histological analyses.

Evaluation of brain edema. Brain edema was evaluated by measuring the brain water content with the wet-dry weight method, as previously described (17). The rats were sacrificed by decapitation under deep anesthesia at 12, 24, 48 and $72 \mathrm{~h}$ following TBI or sham surgery. The brains were removed immediately and weighed with a chemical balance to obtain the wet weight (WW), and then dried at $100^{\circ} \mathrm{C}$ for $24 \mathrm{~h}$ to obtain the dry weight (DW). The percentage of water in the tissues was calculated according to the following formula: $\%$ brain water $=[(\mathrm{WW}-\mathrm{DW}) / \mathrm{WW}] \times 100$.

Morris water maze (MWM) test. The spatial learning ability of rats was assessed in a MWM. The apparatus consisted of a circular black-colored water tank $(180 \mathrm{~cm}$ diameter; $50 \mathrm{~cm}$ high) filled with water $\left(26^{\circ} \mathrm{C}\right)$ to $30-\mathrm{cm}$ depth and virtually divided into four equivalent quadrants: north $(\mathrm{N})$, west $(\mathrm{W})$, south (S) and east (E). A 2-cm submerged escape platform (diameter $12 \mathrm{~cm}$, height $28 \mathrm{~cm}$, made opaque with paint) was placed in the middle of one of the quadrants equidistant from the side wall and the center of the pool. All the rats had been trained to find the platform prior to TBI or the sham operation. For each trial, the rat was randomly placed into a quadrant start point $(\mathrm{N}, \mathrm{S}, \mathrm{E}$ or $\mathrm{W})$ facing the wall of the pool and was allowed a maximum of $60 \mathrm{sec}$ to escape to the platform. The rats that failed to escape within $90 \mathrm{sec}$ were placed on the platform for a maximum of $20 \mathrm{sec}$ and returned to the cage for a new trial (intertrial interval, $10 \mathrm{~min}$ ). Maze performance was recorded using a video camera suspended above the maze and interfaced with a video tracking system (HVS Imaging, Hampton, UK). The average escape latency of a total of four trials was calculated. This test was conducted at 3, 4 and 5 days following trauma or sham operation. 
Modified neurological severity score (NSS). Neurological deficits were evaluated using the NSS, which tests reflexes, alertness, coordination and motor ability. One point is awarded for failure to perform a particular task; thus, a score of ten reflects maximal impairment, whereas a normal rat scores zero. Post-injury, NSS was evaluated at days 1-5. Each animal was assessed by an observer who was blinded to the type of treatment the animal had received.

Histological analysis. The brain tissues were fixed in $4 \%$ paraformaldehyde solution for $24 \mathrm{~h}$, washed with running water for $4 \mathrm{~h}$, then dehydrated with graded alcohol and embedded in paraffin following standard histological procedures. The tissues were serially sectioned at a thickness of $5 \mu \mathrm{m}$. All sections were mounted on glass slides and then stained with hematoxylin and eosin (H\&E). The brain sections of the hippocampus area at roughly $1.9 \mathrm{~mm}$ posterior to the bregma from each animal were used for analysis, and the pyramidal cell density $(\mathrm{cell} / \mathrm{s} / \mathrm{mm})$ in the $\mathrm{CA} 1$ area counted after $\mathrm{H} \& \mathrm{E}$ staining.

Immunofluorescence analysis. The brain tissues were fixed in $4 \%$ paraformaldehyde for $24 \mathrm{~h}$, placed in $30 \%$ sucrose solution (0.1 M PBS, pH 7.4) until they sank to the bottom and then embedded in optimal cutting temperature (OCT) compound. The brain was cut into $15-\mu \mathrm{m}$ thick sections coronally from the anterior to the posterior hippocampus (bregma -1.90 to -3.00 ) using a frozen slicer. The frozen sections were treated with 0.4\% Triton X-100 for $30 \mathrm{~min}$, and blocked in normal donkey serum for $1 \mathrm{~h}$. For double labeling, the sections were incubated with a mixture of rabbit anti-LC3 polyclonal antibody (diluted 1:100; PD014; MBL International Co., Woburn, MA, USA) and mouse anti-NeuN monoclonal antibody (diluted 1:100; MAB377; Millipore Corp., Billerica, MA, USA), or goat anti-TLR4 polyclonal antibody (diluted 1:100; sc-16240; Santa Cruz Biotechnology, Inc., Santa Cruz, CA, USA) and mouse anti-NeuN monoclonal antibody (diluted 1:100; Millipore Corp.) overnight at $4^{\circ} \mathrm{C}$. The following day, the sections were incubated with a mixture of donkey anti-rabbit Alexa-Fluor 594 and donkey anti-mouse Alexa-Fluor 488 or donkey anti-goat Alexa-Fluor 594 and donkey anti-mouse Alexa-Fluor 488 (diluted 1:200; Santa Cruz Biotechnology, Inc.) for $2 \mathrm{~h}$ at $37^{\circ} \mathrm{C}$ in the dark. All cell nuclei were counterstained with 4',6-diamidino2-phenylindole (DAPI). The images were captured under a fluorescence microscope (Olympus FluoView ${ }^{\mathrm{TM}}$ FV1000; Olympus, Tokyo, Japan). The primary antibodies were replaced with PBS in the negative control group. Cell counting of positive cells was undertaken as mentioned above.

Western blot analysis. Briefly, the rats were deeply anesthetized with $10 \%$ chloral hydrate and decapitated. The brains were quickly removed and the hippocampal tissues were dissected on ice. Total proteins were extracted and the protein concentration was determined using a BCA kit (Solarbio, Beijing, China). The samples were subjected to sodium dodecyl sulfate-polyacrylamide gel electrophoresis (SDS-PAGE). Separated proteins on the gel were subsequently transferred onto polyvinylidene fluoride membranes (Roche Diagnostics GmbH, Mannheim, Germany) by a transfer apparatus at $200 \mathrm{~mA}$ for $50 \mathrm{~min}$. The blots were then blocked with 5\% fat-free dry milk for $2 \mathrm{~h}$ at room temperature. Subsequently, the blots were incubated with

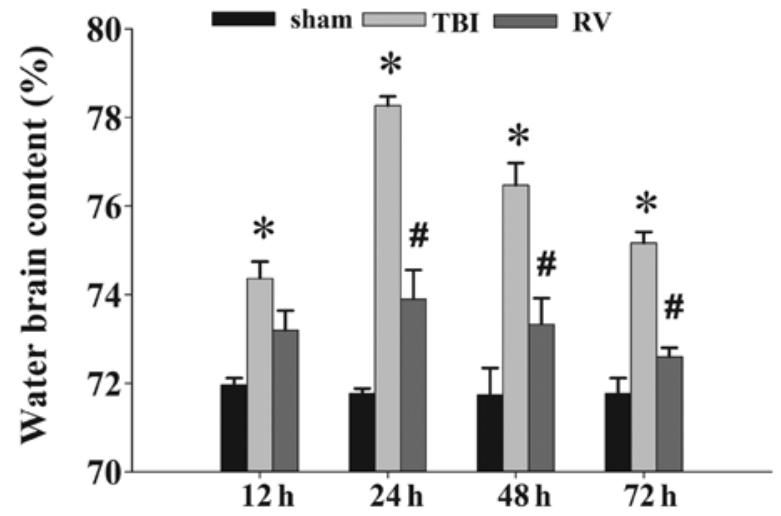

Figure 1. Resveratrol (RV) treatment attenuates brain edema. Brain water content increased markedly at $12,24,48$ and $72 \mathrm{~h}$ following traumatic brain injury (TBI) $\left({ }^{*} \mathrm{P}<0.01\right.$ vs. sham group). Treatment with RV significantly decreased brain edema at $24-72 \mathrm{~h}\left({ }^{\#} \mathrm{P}<0.05\right.$ vs. TBI group), as reflected by a decrease in brain water content. Bars represent the means $\pm \mathrm{SD}$ ( $\mathrm{n}=5 /$ group).

the indicated primary antibodies overnight at $4{ }^{\circ} \mathrm{C}$, including goat anti-TLR4 polyclonal antibody (dilution, 1:500), mouse anti-NF-кB p65 polyclonal antibody (dilution, 1:500) (both from Santa Cruz Biotechnology, Inc.), rabbit anti-tumor necrosis factor (TNF)- $\alpha$ polyclonal antibody (dilution, 1:500; AF7014), rabbit anti-IL-1 $\beta$ polyclonal antibody (dilution, 1:500; AF5103) (both from Affinity Biosciences, Inc., Cincinnati, OH, USA), rabbit anti-LC3 polyclonal antibody (dilution, 1:1,000), rabbit anti-Beclin1 polyclonal antibody (dilution, 1:1,000) (both from MBL International Co.), and rabbit anti- $\beta$-actin monoclonal antibody (dilution, 1:1,000; Affinity Biosciences, Inc.). They were then incubated with horseradish peroxidase conjugated anti-rabbit IgG (1:5,000 dilution), anti-mouse IgG (1:2,000 dilution) and anti-goat IgG (1:1,000 dilution) (all from Santa Cruz Biotechnology, Inc.) secondary antibodies for $2 \mathrm{~h}$ at room temperature. The immunoblot on the membrane was then visible following development with an enhanced chemiluminescence (ECL) detection system, and the densitometric signals were quantified using an imaging program (Image Lab 4.1; Bio-Rad, Berkeley, CA, USA). The intensity of the immunoreactive bands was normalized to the intensity of the corresponding bands for $\beta$-actin. The results were analyzed using the National Institutes of Health Image 1.41 software (Bethesda, MD, USA).

Statistical analysis. Statistical analysis was performed using SPSS 16.0 statistics software (SPSS, Inc., Chicago, IL, USA). Data are expressed as the means \pm standard deviation (SD). Statistical analysis was performed using ANOVA, followed by the Student-Newman Keuls post hoc tests. A P-value of $<0.05$ was considered to indicate a statistically significant difference.

\section{Results}

$R V$ treatment ameliorates brain edema. The wet-dry weight method was used to evaluate brain edema in rats. As is shown in Fig. 1, TBI induced a marked increase in water content at $12,24,48$ and $72 \mathrm{~h}$ in the TBI group compared with the control sham-operated group. However, brain water content in the RV treatment group was significantly reduced at 24,48 and $72 \mathrm{~h}$ compared with that in the TBI group at the same time points. 


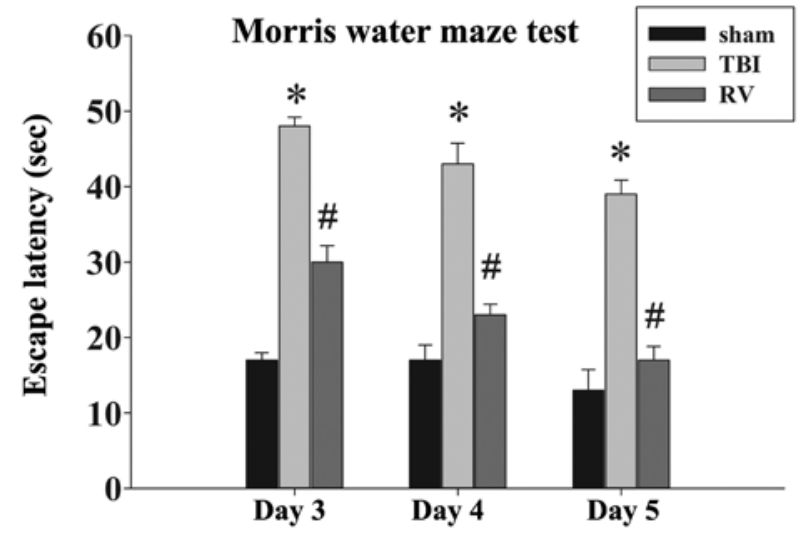

Figure 2. Resveratrol (RV) treatment improves the learning and memory ability. The escape latency increased markedly at 3,4 and 5 days after traumatic brain injury (TBI) ("P<0.01 vs. sham group). Treatment with RV reduced the time to identify the platform $\left({ }^{\#} \mathrm{P}<0.05\right.$ vs. TBI group). Bars represent the means $\pm \operatorname{SD}$ ( $n=5 /$ group).

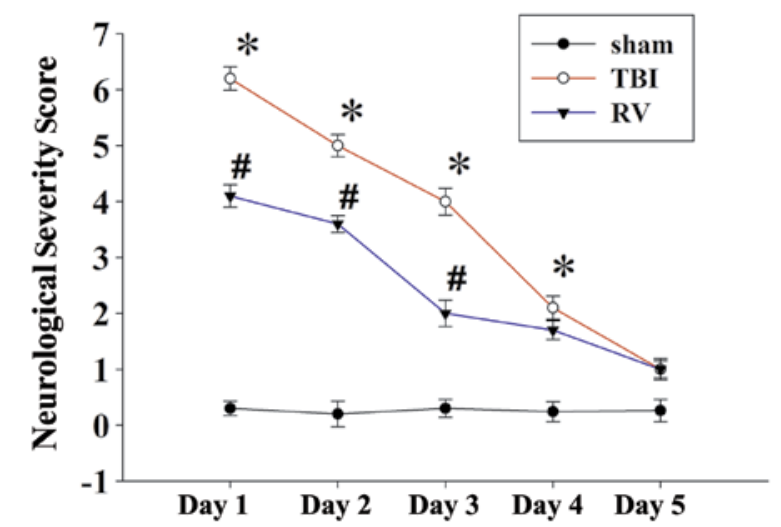

Figure 3. Resveratrol (RV) treatment improves motor function. The neurologic severity score (NSS) of rats was significantly increased at days 1-4 after traumatic brain injury (TBI) ( $\mathrm{P}<0.01$ vs. sham group), and administration of RV significantly improved motor function at days $1-3\left({ }^{*} \mathrm{P}<0.05\right.$ vs. TBI group), as reflected by a decrease in NSS. Bars represent the means \pm SD ( $\mathrm{n}=5 /$ group).

$R V$ treatment improves learning and memory ability as well as motor function. As RV treatment attenuated brain edema, we next examined the effects of RV on the learning and memory ability of rats in a MWM, as well as motor deficit, which were evaluated using the NSS score after TBI. As shown in Fig. 2, TBI caused a significant spatial learning deficit compared with the rats in the sham group, and the administration of RV significantly reduced escape latency at 3,4 and 5 days compared with that in the TBI group. Furthermore, as demonstrated in Fig. 3, the NSS scores of the rats in the TBI group were significantly increased in comparison with the sham group at days 1-4, and RV treatment significantly improved the motor function recovery of the rats with TBI at days 1-3 following TBI.

$R V$ treatment ameliorates hippocampal neuronal damage. $\mathrm{H} \& \mathrm{E}$ staining was performed to examine the effect of RV on hippocampal neuronal damage $24 \mathrm{~h}$ after TBI. In the rats with TBI, there were marked morphological changes in the hippocampal field compared with the rats in the shamoperated group (Fig. 4A), as well as significant neuronal
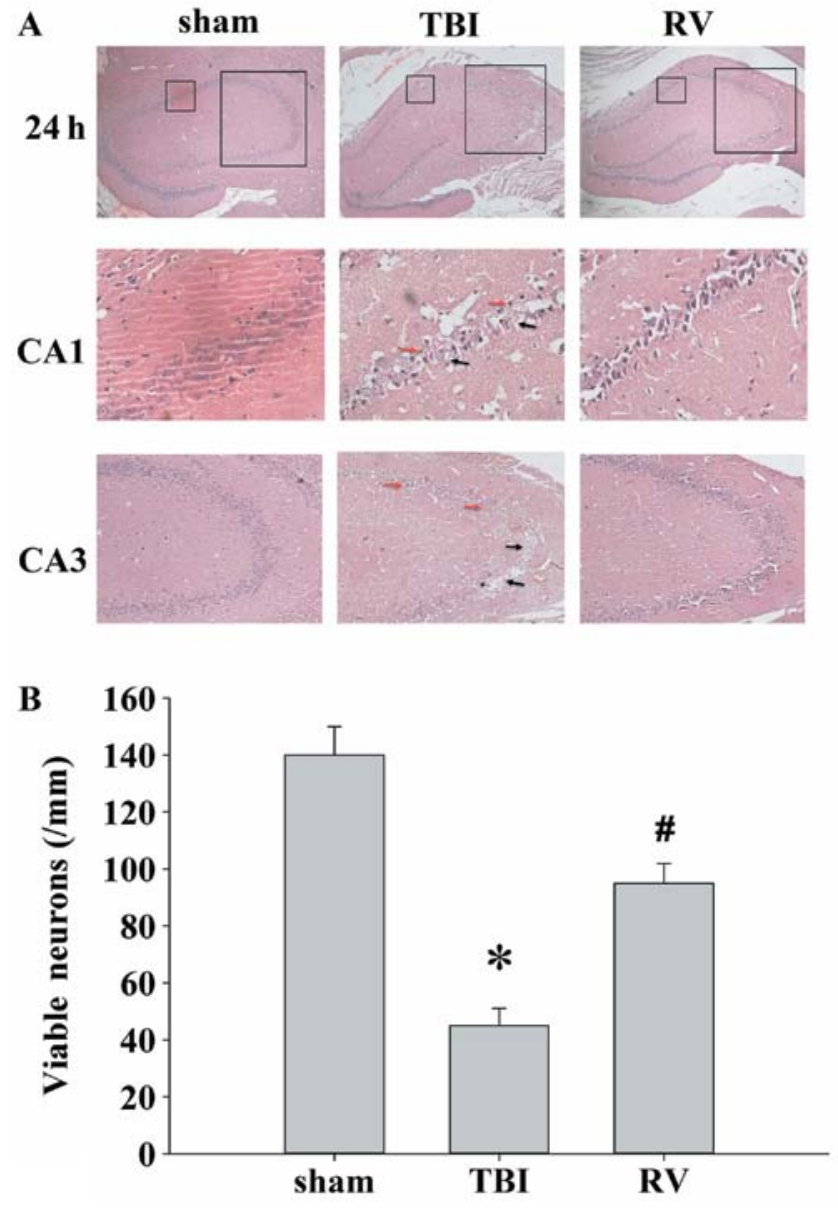

Figure 4. Effect of resveratrol (RV) on hippocampal neuronal damage. (A) Representative H\&E staining in hippocampal areas are shown in the sham-operated, traumatic brain injury (TBI) and RV treatment groups at $24 \mathrm{~h}$ after injury (magnification, $\mathrm{x} 40$ ). Boxed areas are shown at a higher magnification in hippocampal CA1 (magnification, $\mathrm{x} 400$ ) and CA3 (magnification, x100) subfields. Red arrows indicate significant shrinkage, minimal cytoplasm; black arrows indicate outright loss of neurons and widespread damage. (B) Quantification of the number of viable neurons $/ \mathrm{mm}$ of hippocampal area in each group. ( $\mathrm{n}=5 /$ group; $\mathrm{P}<0.01$ vs. sham-operated group, ${ }^{\#} \mathrm{P}<0.05$ vs. TBI group).

damage (Fig. 4B). Microphotographs of the hippocampal CA1 and CA3 subfields for each group are shown in Fig. 4 A. Histological observation revealed that the neurons are clear and of moderate size, with normal ultrastructure, in the shamoperated rats. Pyramidal neurons exhibited either significant shrinkage, minimal cytoplasm or outright loss of neurons and widespread damage in the CA1 and CA 3 subfields in the TBI group after $24 \mathrm{~h}$. Treatment with RV significantly moderated the morphological changes and reduced neuronal loss induced by injury.

RVtreatmentsuppressesneuronalautophagyinthe hippocampus. To further elucidate the roles of RV in the attenuation of TBI-induced cell death and in neuroprotection, we first examined the autophagy marker protein, LC3, in hippocampal neurons $24 \mathrm{~h}$ after TBI using immunofluorescence. As shown in Fig. 5, LC3 was stained with rabbit anti-LC3 antibody and secondary antibodies labeled with red fluorescence; neurons were stained with mouse anti-NeuN antibody and secondary antibody labeled with green fluorescence; cell nuclei were counterstained by 

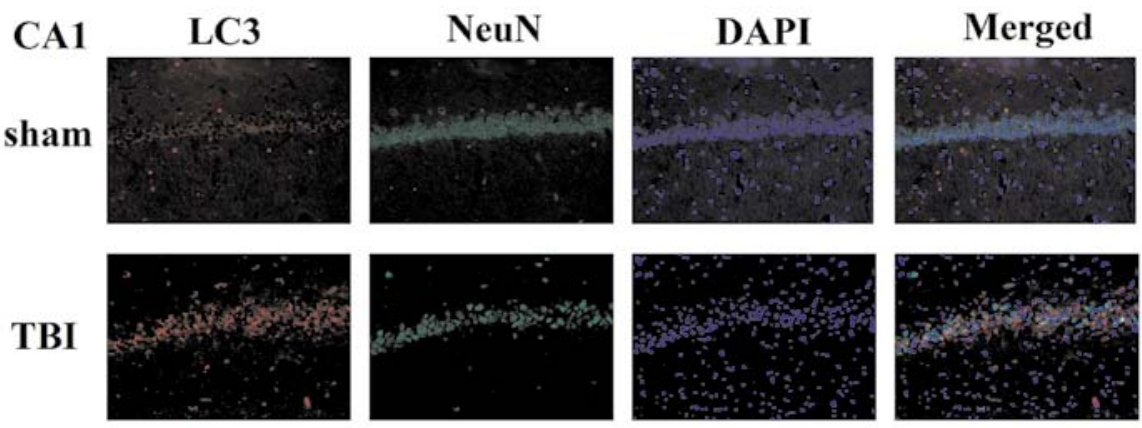

RV
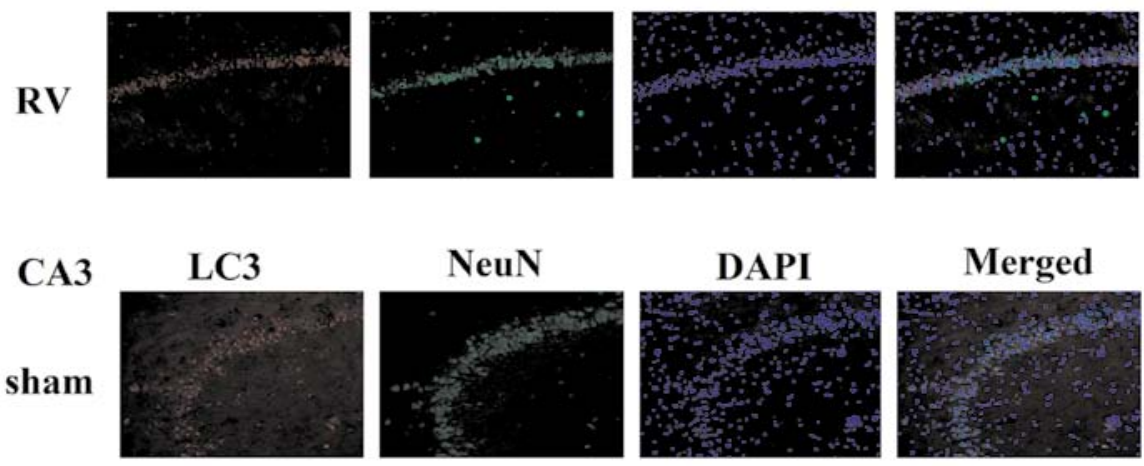

TBI
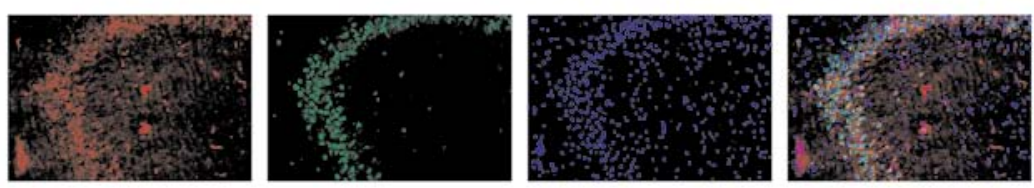

RV
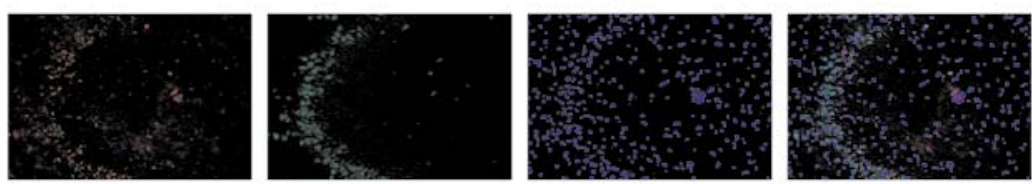

Figure 5. Co-localization of microtubule-associated protein 1 light chain 3 (LC3) and neuron-specific nuclear protein (NeuN) at $24 \mathrm{~h}$ following traumatic brain injury (TBI) was determined by immunofluorescent staining (magnification, x200). As shown, few LC3-positive NeuN cells were detected in the hippocampal CA1 and CA3 subfields from the sham group. The majority of LC3-positive NeuN cells were found in the hippocampal CA1 and CA3 subfields following TBI. Treatment with resveratrol (RV) significantly inhibited the increase in LC3-positive NeuN post-trauma. Orange labeling is indicative of co-localization.
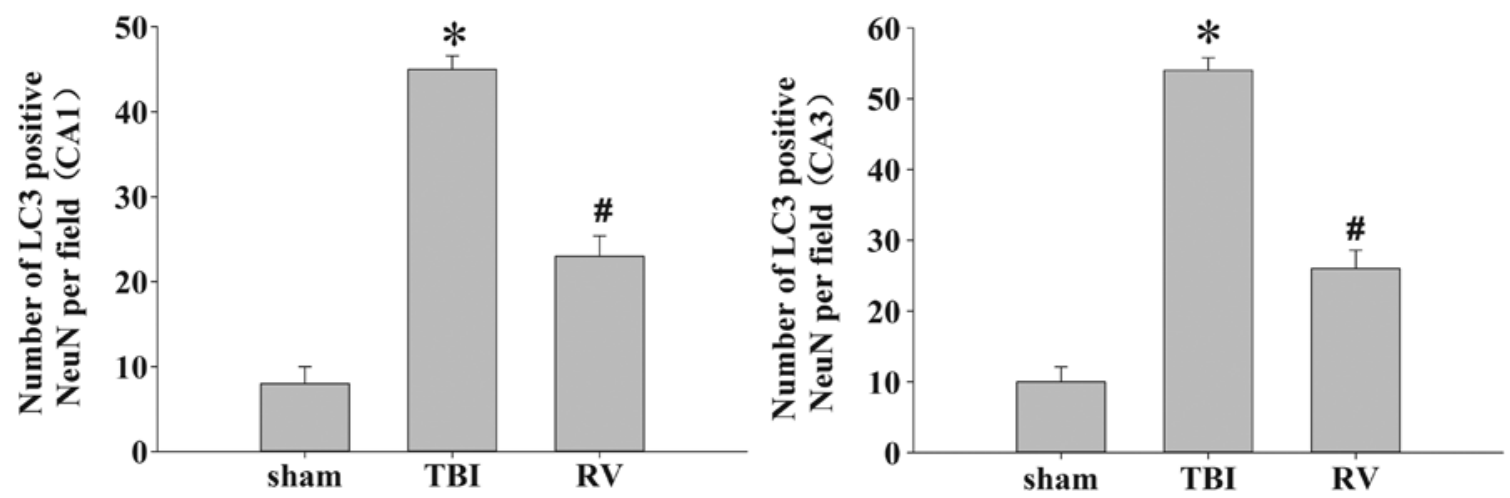

Figure 6. Quantification of microtubule-associated protein 1 light chain 3 (LC3)-positive neuron-specific nuclear protein (NeuN) cells in the hippocampal CA1 and CA3 subfields. The resveratrol (RV)-treated rats had significantly fewer NeuN-positive cells co-labeled with LC3 in hippocampal CA1 and CA3 subfields than the rats in the traumatic brain injury (TBI) group. The total number of NeuN-positive cells co-labeled with LC3 is expressed as the mean number per field of view. ( $\mathrm{n}=5$ /group; ${ }^{*} \mathrm{P}<0.01$ vs. sham-operated group, ${ }^{\text {}} \mathrm{P}<0.05$ vs. TBI group).

DAPI. The images were merged and few LC3-positive NeuN cells were detected in the sham group, while the majority of LC3-positive NeuN cells were found in the hippocampal CA1 and CA3 subfields following TBI $(\mathrm{P}<0.01)$ (Fig. 6). These results showed that the autophagy induced mostly occurred in hippocampal neurons following TBI. Moreover, treatment with RV significantly inhibited the increase in LC3-positive NeuN cells post-trauma $(\mathrm{P}<0.05)$ (Fig. 6).

In order to confirm the ability of RV to inhibit autophagy, the protein levels of LC3II/I and Beclin1 were evaluated by 

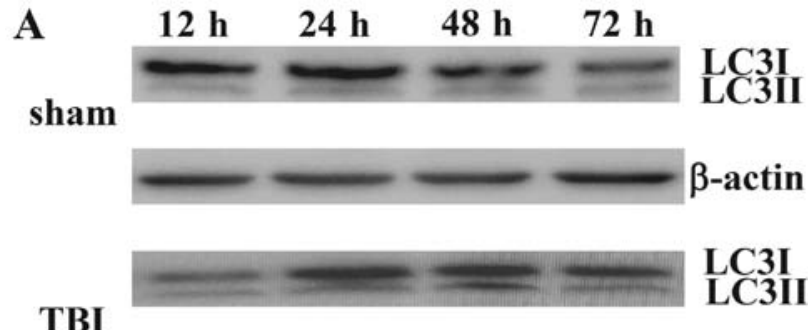

TBI
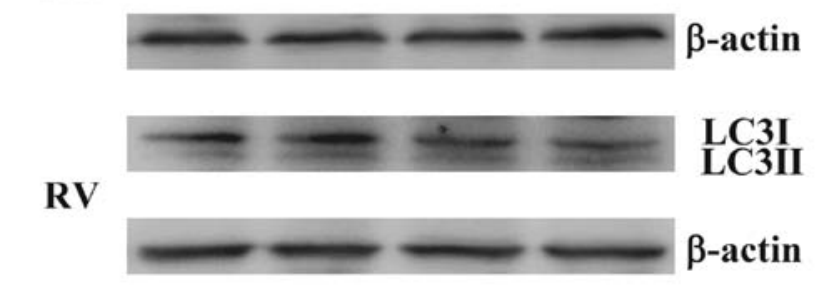

B

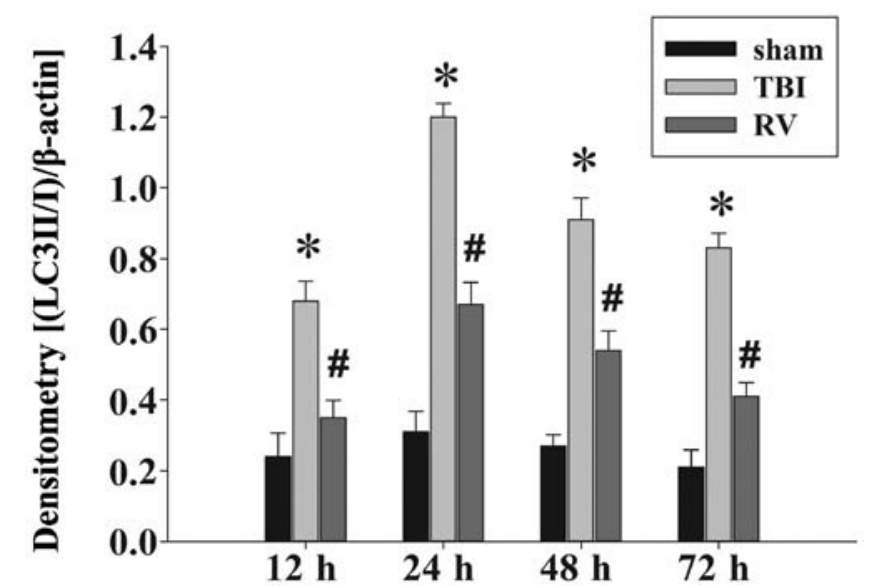

Figure 7. Evaluation of microtubule-associated protein 1 light chain 3 (LC3)II/I protein expression in the hippocampus. (A) Western blot analysis indicates levels of LC3II/I and $\beta$-actin in the hippocampus at $12-72 \mathrm{~h}$ following traumatic brain injury (TBI). (B) Densitometric analysis of LC3II/I expression and $\beta$-actin. The results demonstrated that administration of resveratrol (RV) significantly decreased LC3-II protein expression at $12-72 \mathrm{~h}$ following TBI (" $\mathrm{P}<0.01$ vs. sham group, $\# \mathrm{P}<0.05$ vs. TBI group). The bars represent the means $\pm \mathrm{SD}$ ( $\mathrm{n}=5$ /group).

western blot analysis. The protein expression of LC3II/I was significantly upregulated at $12,24,48$ and $72 \mathrm{~h}$ after TBI compared with that of the sham-operated group. However, compared with the TBI groups, treatment with RV significantly decreased LC3II/I protein expression in the injured hippocampus (Fig. 7). Upregulation of Beclin1 at 12, 24, 48 and $72 \mathrm{~h}$ was observed post-TBI; however, treatment with $\mathrm{RV}$ resulted in a marked decreased in Beclin1 protein levels from 12-72 $\mathrm{h}$ after TBI in the injured hippocampus (Fig. 8), as compared with the TBI group.

$R V$ treatment suppresses TLR4 expression in the hippocampus. To determine whether TLR4 was involved in TBI-induced neuronal damage, we performed double-immunofluorescence staining at $24 \mathrm{~h}$ after TBI. As is clearly shown in Fig. 9A, few NeuN-positive cells co-labeled with TLR4 were detected in the sham-operated group, but the majority of TLR4-positive NeuN cells were found in the hippocampal region CA1 at $24 \mathrm{~h}$ following TBI. RV significantly reduced the number of NeuN-positive cells co-labeled with TLR4 after TBI $(\mathrm{P}<0.05)$ (Fig. 9B). The
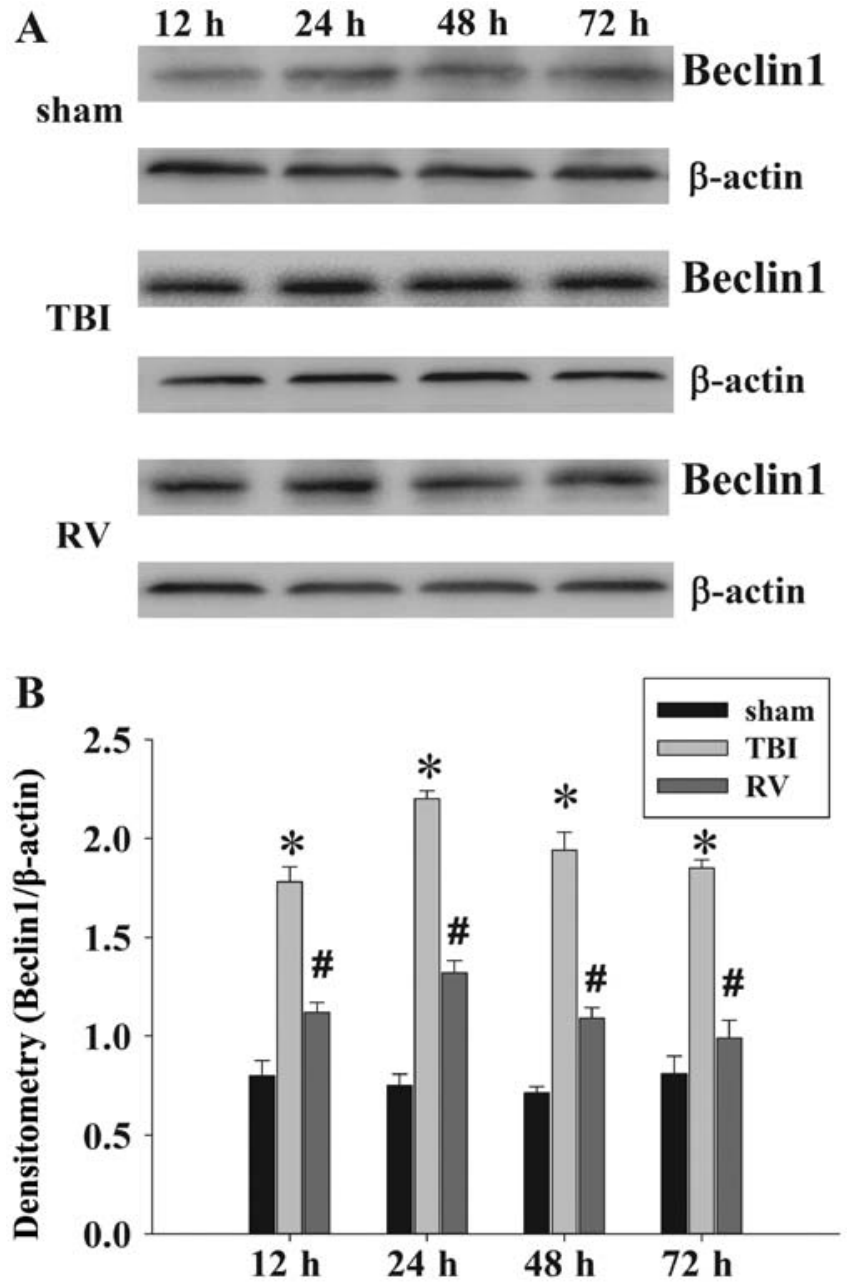

Figure 8. Evaluation of Beclin1 protein expression in the hippocampus (A) Western blot analysis demonstrates levels of Beclin1 and $\beta$-actin in the hippocampus at $12-72 \mathrm{~h}$ following traumatic brain injury (TBI) (B) Densitometric analysis of Beclin1 expression correlated with $\beta$-actin. The results demonstrated that administration of resveratrol (RV) significantly decreased Beclin1 protein expression at $12-72 \mathrm{~h}$ following TBI $\left({ }^{*} \mathrm{P}<0.01\right.$ vs. sham group, ${ }^{\#} \mathrm{P}<0.05$ vs. TBI group). The bars represent the means $\pm \mathrm{SD}$ ( $\mathrm{n}=5$ /group).

protein levels of TLR4 were subsequently analyzed by western blot analysis (Fig. 10). The expression of TLR4 was identified at low levels in the hippocampus in the sham-operated group, and it was significantly increased at 12, 24, 48 and $72 \mathrm{~h}$ after injury, and reached a maximum level at $24 \mathrm{~h}$. However, RV significantly inhibited the upregulation of TLR4 protein levels, as compared with the TBI groups at 12-72 h.

$R V$ treatment significantly downregulates the expression of $N F-\kappa B$ p65 and inflammatory cytokines in the hippocampus. To investigate the mechanism of action of RV further, western blot analysis was performed to examine the expression of $\mathrm{NF}-\kappa \mathrm{B}$ p65 and inflammatory cytokines in the hippocampus. The results showed that the expression levels of NF- $\mathrm{B}$ p65 were significantly upregulated in the hippocampal tissues of rats in the TBI group compared with the sham group at 12, 24, 48 and $72 \mathrm{~h}$ following TBI. Compared with the TBI group, the expression levels of NF- $\kappa \mathrm{B}$ p65 were significantly lower in the hippocampal tissues of rat in the RV group at 12-72 $\mathrm{h}$ after TBI (Fig. 11). We then performed western blot anlaysis to deter- 

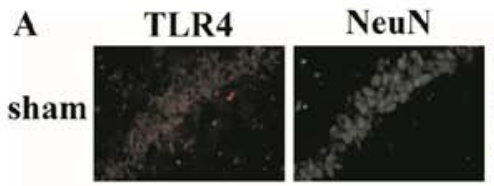

TBI
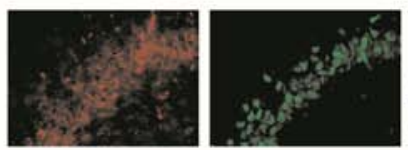

RV

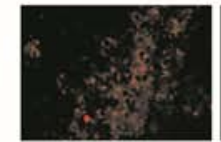

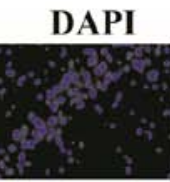
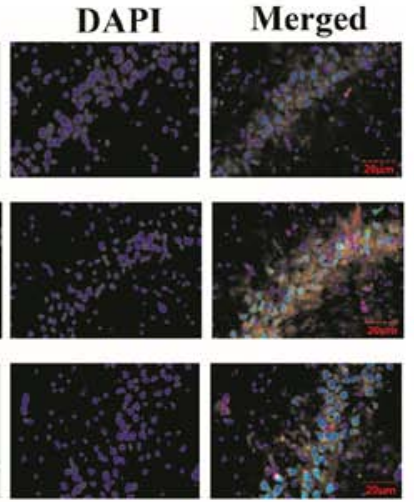

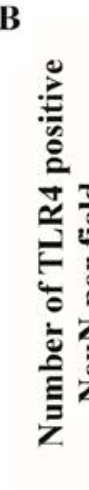

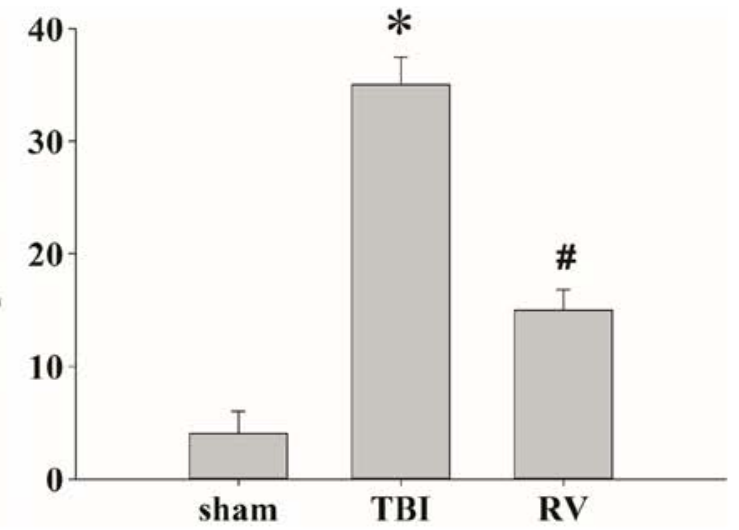

Figure 9. (A) Identification of Toll-like receptor (TLR)4-positive cells 24 h post-traumatic brain injury (TBI) in the hippocampus using immunofluorescence (magnification, x400). TLR4 immunoreactivity (red) was present in neuron-specific nuclear protein (NeuN)-positive cells (green) at $24 \mathrm{~h}$ following TBI, and cell nuclei were counterstained by DAPI (blue). Orange labeling indicates co-localization. Scale bar, $20 \mu \mathrm{m}$. (B) Quantification showing that resveratrol (RV)-treated rats had significantly fewer NeuN-positive cells co-labeled with TLR4 in the hippocampal area than the TBI group. The total number of NeuN-positive cells co-labeled with TLR4 is expressed as the mean number/field of view. ( $\mathrm{n}=5 /$ group; ${ }^{*} \mathrm{P}<0.01 \mathrm{vs}$. sham group, ${ }^{\text {"}} \mathrm{P}<0.05$ vs. TBI group).

A

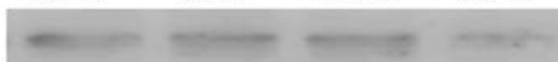

sham

sham

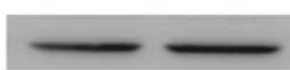

TBI

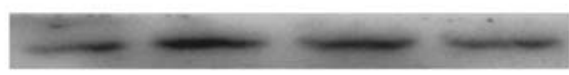

\section{TLR4}

$\beta$-actin

TLR4

RV

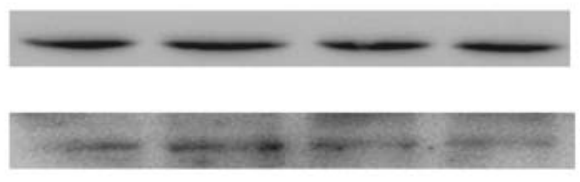

$\beta$-actin

B
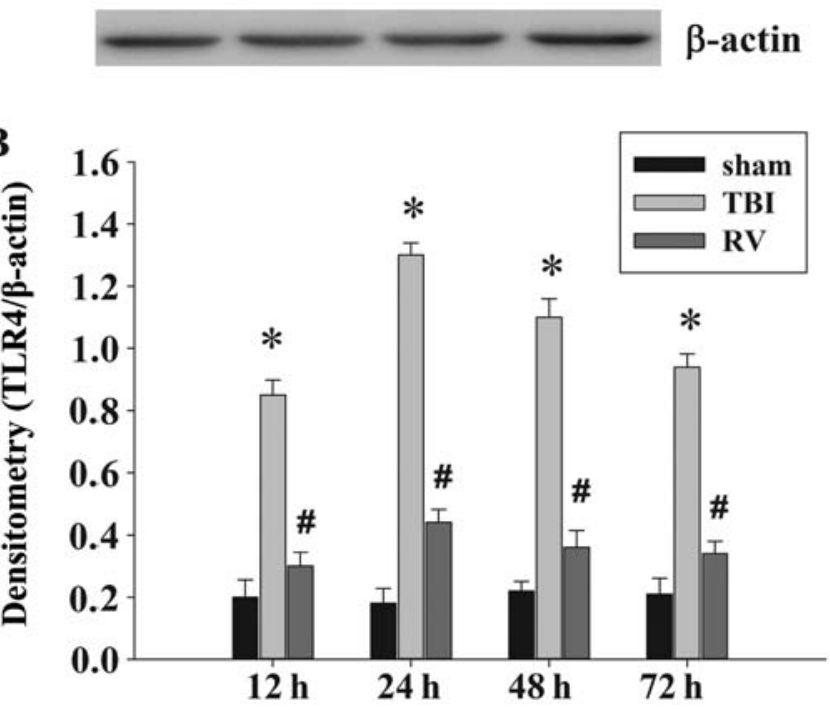

Figure 10. Evaluation of Toll-like receptor (TLR)4 protein expression in the hippocampus. (A) Western blot analysis demonstrates the levels of TLR4, normalized to $\beta$-actin, in the hippocampus at $12,24,48$ and $72 \mathrm{~h}$ following traumatic brain injury (TBI). (B) Densitometric analysis of TLR4 expression correlated with $\beta$-actin. The results demonstrated that TLR4 protein expression was significantly elevated at $12-72 \mathrm{~h}$ following TBI $\left({ }^{*} \mathrm{P}<0.01\right.$ vs. sham group). Administration of resveratrol (RV) significantly decreased the level of TLR4 ( ${ }^{\#} \mathrm{P}<0.05$ vs. TBI group). Data are expressed as the means \pm SD ( $n=5 /$ group). mine the expression levels of inflammatory factors, including IL-1 $\beta$ and TNF- $\alpha$, in the hippocampal tissues of rats following TBI. As shown in Fig. 12, the levels of IL-1 $\beta$ and TNF- $\alpha$ were significantly increased in the TBI group compared with those in the sham-operated group at 12-72 $\mathrm{h}$ after TBI, and both of these were significantly reduced by RV. These results indicate that RV significantly reduces the production of inflammatory factors in hippocampal tissues in a rat model of TBI.

\section{Discussion}

$\mathrm{RV}$ is undergoing extensive investigation for its multifaceted properties. In particular, experimental evidence has demonstrated that RV exerts a neuroprotective effect in a variety of models of brain injury $(18,20,25-27)$. However, the association between the neuroprotective effect of RV and signal molecules in the brain injury remains to be elucidated. The present study used a rat model of TBI caused by CCI and indicated that the administration of RV to rats following TBI may exert a neuroprotective effect, effected through the TLR4-mediated signaling pathway. To the best of our knowledge, we report for the first time a possible molecular mechanism through which RV attenuates brain injury. It involves the modulation of neuronal autophagy and acute neuroinflammation which is mediated by the TLR4/NF- $\mathrm{B}$ signaling pathway in the hippocampus of rat brains damaged by experimental TBI.

TBI is caused by immediate brain tissue disruption (primary injury) and post-injury cellular and molecular events (secondary injury); these include inflammation, mitochondrial dysfunction, oxidative stress, excitotoxic damage and increased vascular permeability $(27,28)$. This combination of cellular and physiologic disturbances causes increased neuronal cell death, as well as neurological, motor and cognitive impairment (29). Thus, we firstly evaluated whether RV attenuates brain edema, and motor and cognitive impairment, following TBI. In the present study, we demonstrated that rats treated with RV $(100 \mathrm{mg} / \mathrm{kg} / \mathrm{day}$, up to 3 days) exhibited reduced post-traumatic brain edema, improved learning and memory ability and reduced motor deficits. These 

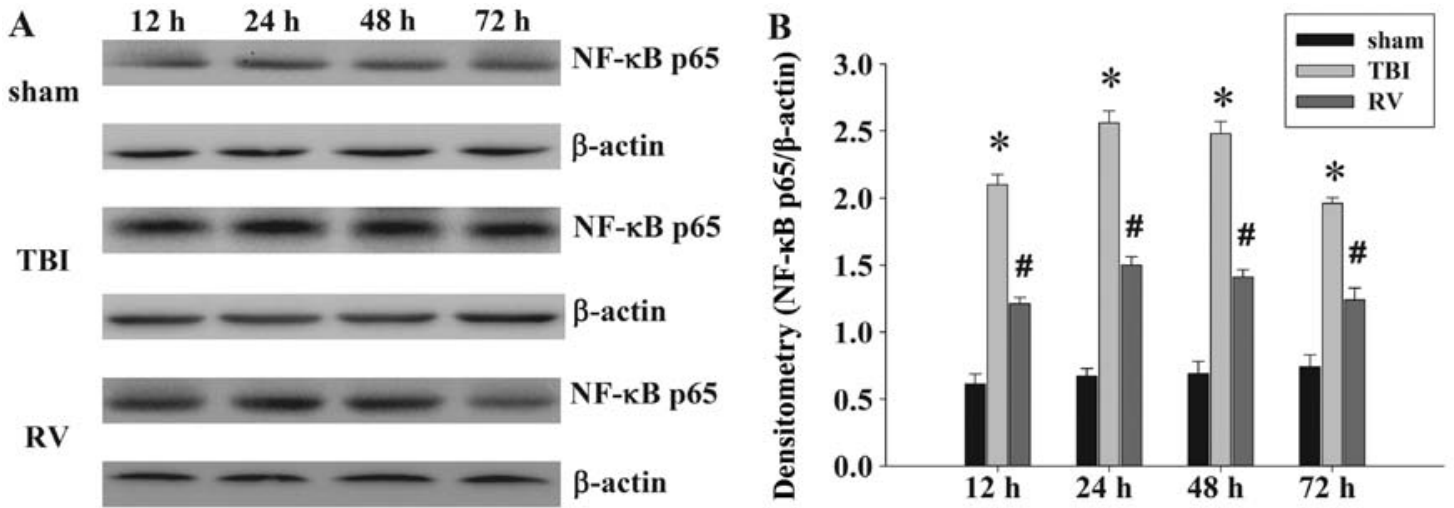

Figure 11. Evaluation of nuclear factor- $\mathrm{kB}(\mathrm{NF}-\mathrm{\kappa B}) \mathrm{p} 65$ protein expression in the hippocampus. (A) Western blot analysis demonstrates levels of NF- $\mathrm{kB}$ p65 and $\beta$-actin in the hippocampus at 12, 24, 48 and $72 \mathrm{~h}$ following traumatic brain injury (TBI). (B) Densitometry of NF- $\mathrm{kB}$ p65 expression correlated with $\beta$-actin. The results demonstrated that NF- $\mathrm{KB}$ p65 expression was significantly elevated at $12-72 \mathrm{~h}$ following TBI ("P $<0.01$ vs. sham group). Administration of resveratrol (RV) significantly decreased the level of NF- $\mathrm{KB}$ p65 (" $\mathrm{P}<0.05$ vs. TBI group). Data are expressed as the means \pm SD ( $\mathrm{n}=5 / \mathrm{group})$.
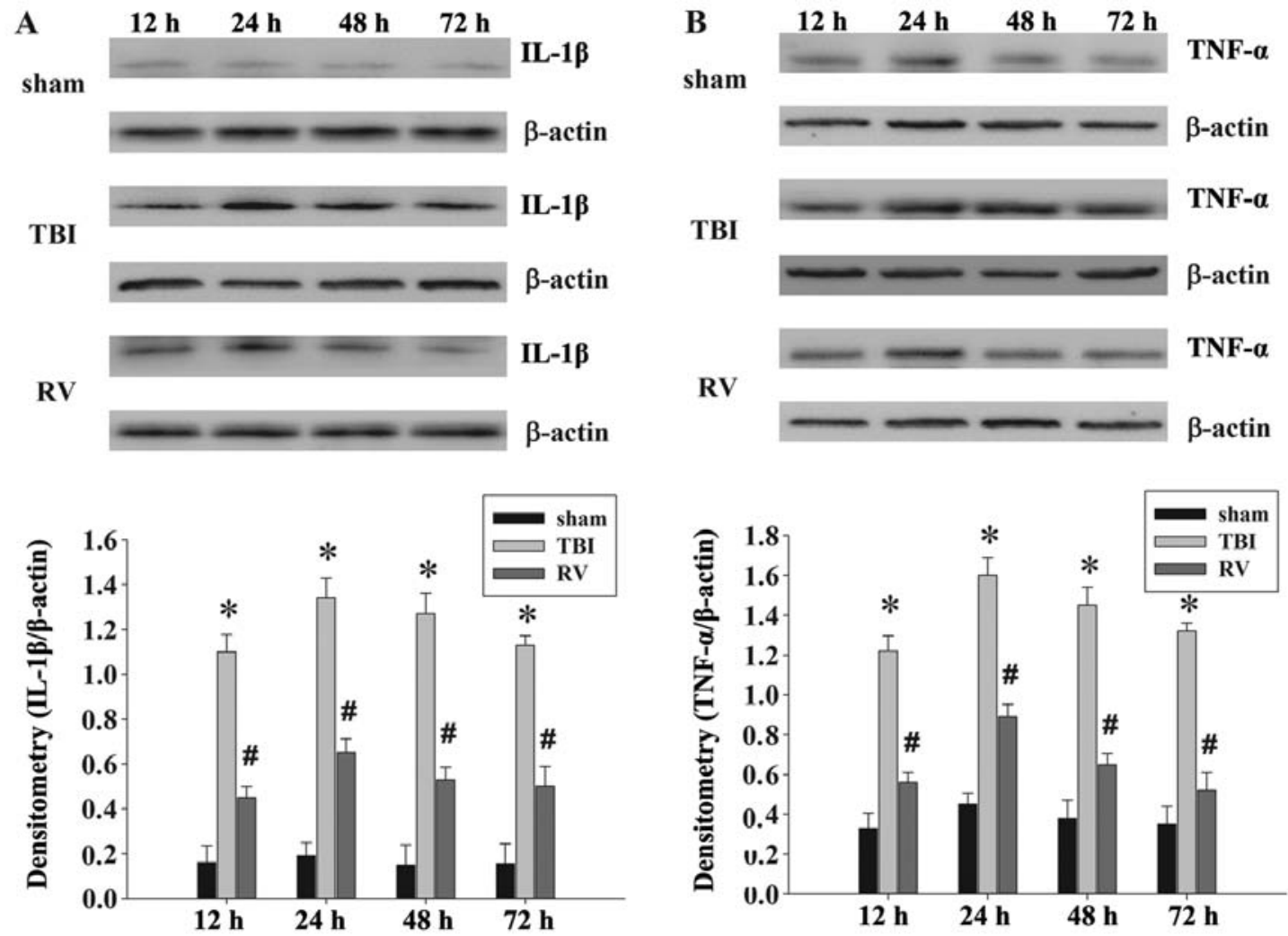

Figure 12. Evaluation of interleukin (IL)-1 $\beta$ and tumor necrosis factor (TNF)- $\alpha$ protein expression in the hippocampus. (A) Western blot analysis demonstrates levels of IL- $1 \beta$ and $\beta$-actin in the hippocampus at 12,24,48 and $72 \mathrm{~h}$ following traumatic brain injury (TBI). (B) Densitometry of TNF- $\alpha$ correlated with the $\beta$-actin band. The results demonstrated that the expression of IL- $1 \beta$ and TNF- $\alpha$ was significantly elevated at $12-72 \mathrm{~h}$ following TBI ("P<0.01 vs. sham group). Administration of resveratrol (RV) significantly decreased the levels of IL- $1 \beta$ and TNF- $\alpha\left({ }^{*} \mathrm{P}<0.05\right.$ vs. TBI group). Data are expressed as the means \pm SD ( $n=5 /$ group).

results are consistent with previous studies suggesting that RV treatment affords neuroprotection against a variety of neurologic disorders, including attenuation of brain edema formation (18) and motor and cognitive impairment $(25,30)$. Subsequently, we evaluated neuronal damage, using H\&E staining, in the hippocampus following TBI, and we found that there were marked morphological changes in the hippocampus CA1 and CA 3 neurons of rats in the TBI group compared with the sham-operated controls, as well as significant neuronal loss.
Treatment with RV significantly moderated the morphologic changes and increased neuronal survival. This result is similar to the findings of Singleton et al, who demonstrated that treatment with RV also selectively protects vulnerable neurons in the CA1 and CA3 regions (30). Lin et al found that the administration of RV increased cell survival by suppressing GSK-3b-mediated autophagy and apoptosis (20), and thus we hypothesized that the mechanism underlying the neuroprotective effects of RV on TBI is mediated through the prevention of autophagic neuronal death. 
In the present study, experiments using in vivo models of TBI showed that the administration of RV suppressed neuronal autophagy in the hippocampus of rat brains. Of note, the role of neuronal autophagy following acute brain injury remains controversial. Sarkar et al showed that autophagic clearance is impaired soon after TBI due to lysosomal dysfunction, and that this correlates with neuronal cell death (31). Lipinski et al found that augmentation and/or restoration of autophagy flux provide a potential therapeutic target for the treatment of TBI and spinal cord injury (32). By contrast, certain studies have demonstrated that autophagy triggers cell injury and death under certain pathological circumstances $(11,33)$. A previous study has suggested that excessive autophagy is a contributing factor to neuronal death in cerebral ischemia and hypoxia (34), and our previous studies have shown that TBI activates autophagy-exacerbated brain injury, which may begin at $6 \mathrm{~h}$ or earlier, and peaks at $24 \mathrm{~h}$ in the hippocampus following TBI $(14,35)$. Thus, our results are in line with the latter findings. However, the involvement of autophagy in the neuroprotective effect of RV warrants furtherinvestigation. Previous studies have demonstrated that a combination of rapamycin and RV blocks rapamycin-induced autophagy but promotes apoptosis of tuberous sclerosis 2 (TSC2)-deficient cells and breast cancer cells $(21,36)$. On the contrary, it has been suggested that RV induces protective autophagy; the data indicate that RV suppresses NLR family, pyrin domain containing 3 (NLRP3) inflammasome activation by preserving mitochondrial integrity and by augmenting autophagy (37). Wang et al have shown that RV improves cardiac dysfunction and remodeling, and attenuates cardiomyocyte apoptosis and oxidative stress injury in diabetic mice through enhanced autophagic flux (38). Therefore, the effect of RV on autophagy likely depends on the environment or the stimuli, and on the cell type or animal model. Our results are in line with those of these previous studies, and it is therefore conceivable to hypothesize that the neuroprotective effect of RV on TBI is associated with the attenuation of neuronal autophagy, which is a contributing factor to neuronal death.

TLR4 is considered to play an important role in neuronal death in animal models and may be an important therapeutic target following TBI. Previous studies have shown that the activation of TLR4 induces NF- $\mathrm{BB}$-dependent apoptosis and expression of proinflammatory cytokines $(39,40)$. Indeed, RV is a potent inhibitor of a wide variety of inflammatory agents which induce the activation of $\mathrm{NF}-\kappa \mathrm{B}$, and this inhibition is not cell-type specific. In order to gain a more in-depth understanding of the molecular mechanisms underlying RV, and the anti-inflammatory effects of RV, we found that RV inhibits the expression of TLR4 and decreases the expression and activity of $\mathrm{NF}-\kappa \mathrm{B}$ p65 in the hippocampus of rat brains, for the first time, to the best of our knowledge. In addition, we have demonstrated that RV also attenuates the release of inflammatory cytokines (IL-1 $\beta$ and TNF- $\alpha$ ) in the hippocampal tissue of rats. Certain molecular targets of RV have been previously identified in the TLR-mediated signaling pathways. Youn et al reported that RV inhibited MyD88-independent signaling pathways and targeted expression through TANK-binding kinase 1 (TBK1) and receptor-interacting protein 1 (RIP1) in the TIR-domaincontaining adapter-inducing interferon- $\beta$ (TRIF) complex (41). Li et al provided evidence that RV inhibits TLR4/NF- $\kappa \mathrm{B}$ signaling in rat hearts subjected to ischaemia/reperfusion, and the anti-inflammatory effect of RV is associated with nitric oxide (NO) production (42). Another study has shown that RV attenuates the inflammatory response in mice with diabetic periodontitis by downregulating TLR4 expression and TLR4 downstream signaling activation (43). Therefore, we suggest that the neuroprotective and anti-inflammatory effects exerted by RV following TBI are associated with the inhibition of the TLR4/NF- $\kappa \mathrm{B}$ signaling pathway.

In conclusion, our data provide new insights into the role played by RV in protecting the brain against TBI. This study indicated that RV attenuates brain edema and improves motor and cognitive functional recovery following TBI. Furthermore, we hypothesized that these protective effects are associated with RV-mediated reduction of neuronal autophagy and inhibition of the TLR4/NF- $\kappa \mathrm{B}$ signaling pathway. It is now necessary to undertake in vitro studies to determine the potentially protective role of RV in the treatment of TBI.

\section{Acknowledgements}

The present study was supported by a grant from the Natural Science Foundation of Hebei Province (grant no. H2014105079).

\section{References}

1. Greve MW and Zink BJ: Pathophysiology of traumatic brain injury. Mt Sinai J Med 76: 97-104, 2009.

2. Helmy A, De Simoni MG, Guilfoyle MR, Carpenter KL and Hutchinson PJ: Cytokines and innate inflammation in the pathogenesis of human traumatic brain injury. Prog Neurobiol 95: 352-372, 2011.

3. Ziebell JM and Morganti-Kossmann MC: Involvement of pro- and anti-inflammatory cytokines and chemokines in the pathophysiology of traumatic brain injury. Neurotherapeutics 7: 22-30, 2010.

4. Kopp E and Medzhitov R: Recognition of microbial infection by Toll-like receptors. Curr Opin Immunol 15: 396-401, 2003.

5. Fang H, Wang PF, Zhou Y, Wang YC and Yang QW: Toll-like receptor 4 signaling in intracerebral hemorrhage-induced inflammation and injury. J Neuroinflammation 10: 27, 2013.

6. Zhu HT, Bian C, Yuan JC, Chu WH, Xiang X, Chen F, Wang CS, Feng $\mathrm{H}$ and Lin JK: Curcumin attenuates acute inflammatory injury by inhibiting the TLR4/MyD88/NF- $\mathrm{BB}$ signaling pathway in experimental traumatic brain injury. J Neuroinflammation 11: 59, 2014.

7. Ahmad A, Crupi R, Campolo M, Genovese T, Esposito E and Cuzzocrea S: Absence of TLR4 reduces neurovascular unit and secondary inflammatory process after traumatic brain injury in mice. PLoS One 8: e57208, 2013.

8. Li GZ,Zhang Y,Zhao JB, Wu GJ, Su XF and Hang CH: Expression of myeloid differentiation primary response protein 88 (Myd88) in the cerebral cortex after experimental traumatic brain injury in rats. Brain Res 1396: 96-104, 2011.

9. Wang X, Stridh L, Li W, Dean J, Elmgren A, Gan L, Eriksson K, Hagberg $\mathrm{H}$ and Mallard C: Lipopolysaccharide sensitizes neonatal hypoxic-ischemic brain injury in a MyD88-dependent manner. J Immunol 183: 7471-7477, 2009.

10. Pozuelo-Rubio M: 14-3-3 $\zeta$ binds class III phosphatidylinositol-3-kinase and inhibits autophagy. Autophagy 7: 240-242, 2011.

11. Bursch W, Hochegger K, Torok L, Marian B, Ellinger A and Hermann RS: Autophagic and apoptotic types of programmed cell death exhibit different fates of cytoskeletal filaments. J Cell Sci 113: 1189-1198, 2000.

12. Luo CL, Li BX, Li QQ, Chen XP, Sun YX, Bao HJ, Dai DK, Shen YW, Xu HF, Ni H, et al: Autophagy is involved in traumatic brain injury-induced cell death and contributes to functional outcome deficits in mice. Neuroscience 184: 54-63, 2011.

13. Bao HJ, Zhang L, Han WC and Dai DK: Apelin-13 attenuates traumatic brain injury-induced damage by suppressing autophagy. Neurochem Res 40: 89-97, 2015. 
14. Cui C, Cui Y, Gao J, Sun L, Wang Y, Wang K, Li R, Tian Y, Song S and Cui J: Neuroprotective effect of ceftriaxone in a rat model of traumatic brain injury. Neurol Sci 35: 695-700, 2014.

15. Xu Y, Jagannath C, Liu XD, Sharafkhaneh A, Kolodziejska KE and Eissa NT: Toll-like receptor 4 is a sensor for autophagy associated with innate immunity. Immunity 27: 135-144, 2007.

16. Seay MD and Dinesh-Kumar SP: Autophagy takes its TOLL on innate immunity. Cell Host Microbe 2: 69-70, 2007.

17. Xu Y, Liu XD, Gong X and Eissa NT: Signaling pathway of autophagy associated with innate immunity. Autophagy 4: 110-112, 2008

18. Ates O, Cayli S, Altinoz E, Gurses I, Yucel N, Sener M, Kocak A and Yologlu S: Neuroprotection by resveratrol against traumatic brain injury in rats. Mol Cell Biochem 294: 137-144, 2007.

19. Gatson JW, Liu MM, Abdelfattah K, Wigginton JG, Smith S, Wolf S and Minei JP: Resveratrol decreases inflammation in the brain of mice with mild traumatic brain injury. J Trauma Acute Care Surg 74: 470-475, 2013.

20. Lin CJ, Chen TH, Yang LY and Shih CM: Resveratrol protects astrocytes against traumatic brain injury through inhibiting apoptotic and autophagic cell death. Cell Death Dis 5: e1147, 2014.

21. Alayev A, Sun Y, Snyder RB, Berger SM, Yu JJ and Holz MK: Resveratrol prevents rapamycin-induced upregulation of autophagy and selectively induces apoptosis in TSC2-deficient cells. Cell Cycle 13: 371-382, 2014.

22. Zhang C, Lin G, Wan W, Li X, Zeng B, Yang B and Huang C: Resveratrol, a polyphenol phytoalexin, protects cardiomyocytes against anoxia/reoxygenation injury via the TLR4/NF- $\mathrm{B}$ signaling pathway. Int J Mol Med 29: 557-563, 2012.

23. Liu L, Gu H, Liu H, Jiao Y, Li K, Zhao Y, An L and Yang J: Protective effect of resveratrol against IL-1 $\beta$-induced inflammatory response on human osteoarthritic chondrocytes partly via the TLR4/MyD88/NF- $\kappa \mathrm{B}$ signaling pathway: an "in vitro study'. Int J Mol Sci 15: 6925-6940, 2014.

24. Mahmood A, Lu D and Chopp M: Marrow stromal cell transplantation after traumatic brain injury promotes cellular proliferation within the brain. Neurosurgery 55: 1185-1193, 2004.

25. Sönmez U, Sönmez A, Erbil G, Tekmen I and Baykara B: Neuroprotective effects of resveratrol against traumatic brain injury in immature rats. Neurosci Lett 420: 133-137, 2007.

26. Shao AW, Wu HJ, Chen S, Ammar AB, Zhang JM and Hong Y: Resveratrol attenuates early brain injury after subarachnoid hemorrhage through inhibition of NF- $\kappa \mathrm{B}$-dependent inflammatory/MMP-9 pathway. CNS Neurosci Ther 20: 182-185, 2014.

27. Leker RR and Shohami E: Cerebral ischemia and traumadifferent etiologies yet similar mechanisms: neuroprotective opportunities. Brain Res Brain Res Rev 39: 55-73, 2002.

28. Bramlett HM and Dietrich WD: Pathophysiology of cerebral ischemia and brain trauma: Similarities and differences. J Cereb Blood Flow Metab 24: 133-150, 2004.

29. Yatsiv I, Grigoriadis N, Simeonidou C, Stahel PF, Schmidt OI, Alexandrovitch AG, Tsenter J and Shohami E: Erythropoietin is neuroprotective, improves functional recovery, and reduces neuronal apoptosis and inflammation in a rodent model of experimental closed head injury. FASEB J 19: 1701-1703, 2005.
30. Singleton RH, Yan HQ, Fellows-Mayle W and Dixon CE: Resveratrol attenuates behavioral impairments and reduces cortical and hippocampal loss in a rat controlled cortical impact model of traumatic brain injury. J Neurotrauma 27: 1091-1099, 2010.

31. Sarkar C, Zhao Z, Aungst S, Sabirzhanov B, Faden AI and Lipinski MM: Impaired autophagy flux is associated with neuronal cell death after traumatic brain injury. Autophagy 10: 2208-2222, 2014

32. Lipinski MM, Wu J, Faden AI and Sarkar C: Function and mechanisms of autophagy in brain and spinal cord trauma. Antioxid Redox Signal 23: 565-577, 2015.

33. Shimizu S, Kanaseki T, Mizushima N, Mizuta T, ArakawaKobayashi S, Thompson CB and Tsujimoto Y: Role of Bcl-2 family proteins in a non-apoptotic programmed cell death dependent on autophagy genes. Nat Cell Biol 6: 1221-1228, 2004.

34. Shi R, Weng J, Zhao L, Li XM, Gao TM and Kong J: Excessive autophagy contributes to neuron death in cerebral ischemia. CNS Neurosci Ther 18: 250-260, 2012.

35. Sun LQ, Gao JL, Cui CM, Cui Y, Jing XB, Zhao MM, Wang YC, Tian YX, Wang KJ and Cui JZ: Astrocytic p-connexin 43 regulates neuronal autophagy in the hippocampus following traumatic brain injury in rats. Mol Med Rep 9: 77-82, 2014

36. Alayev A, Berger SM, Kramer MY, Schwartz NS and Holz MK: The combination of rapamycin and resveratrol blocks autophagy and induces apoptosis in breast cancer cells. J Cell Biochem 116: 450-457, 2015.

37. Chang YP, Ka SM, Hsu WH, Chen A, Chao LK, Lin CC, Hsieh CC, Chen MC, Chiu HW, Ho CL, et al: Resveratrol inhibits NLRP3 inflammasome activation by preserving mitochondrial integrity and augmenting autophagy. J Cell Physiol 230: 1567-1579, 2015.

38. Wang B, Yang Q, Sun YY, Xing YF, Wang YB, Lu XT, Bai WW, Liu XQ and Zhao YX: Resveratrol-enhanced autophagic flux ameliorates myocardial oxidative stress injury in diabetic mice. J Cell Mol Med 18: 1599-1611, 2014.

39. Han KJ, Su X, Xu LG, Bin LH,Zhang J and Shu HB: Mechanisms of the TRIF-induced interferon-stimulated response element and NF-kappaB activation and apoptosis pathways. J Biol Chem 279: 15652-15661, 2004.

40. Baumgarten G, Knuefermann P, Nozaki N, Sivasubramanian N, Mann DL and Vallejo JG: In vivo expression of proinflammatory mediators in the adult heart after endotoxin administration: the role of Toll-like receptor-4. J Infect Dis 183: 1617-1624, 2001.

41. Youn HS, Lee JY, Fitzgerald KA, Young HA, Akira S and Hwang DH: Specific inhibition of MyD88-independent signaling pathways of TLR3 and TLR4 by resveratrol: molecular targets are TBK1 and RIP1 in TRIF complex. J Immunol 175: 3339-3346, 2005.

42. Li J, Xie C, Zhuang J, Li H, Yao Y, Shao C and Wang H: Resveratrol attenuates inflammation in the rat heart subjected to ischemia-reperfusion: role of the TLR4/NF- $\mathrm{B}$ signaling pathway. Mol Med Rep 11: 1120-1126, 2015.

43. Zhen L, Fan DS, Zhang Y, Cao XM and Wang LM: Resveratrol ameliorates experimental periodontitis in diabetic mice through negative regulation of TLR4 signaling. Acta Pharmacol Sin 36: 221-228, 2015. 